\title{
BMJ Open Residential proximity to major roads, exposure to fine particulate matter and aortic calcium: the Framingham Heart Study, a cohort study
}

Kirsten S Dorans, ${ }^{1,2}$ Elissa H Wilker, ${ }^{1,2,3}$ Wenyuan Li, ${ }^{1,2}$ Mary B Rice, ${ }^{2,4}$ Petter L Ljungman,, ${ }^{2,5}$ Joel Schwartz, ${ }^{1,3}$ Brent A Coull, ${ }^{3,6}$ Itai Kloog, ${ }^{7}$ Petros Koutrakis, ${ }^{3}$ Ralph B D'Agostino, ${ }^{8,9}$ Joseph M Massaro, ${ }^{8,10}$ Udo Hoffmann, ${ }^{11}$ Christopher J O'Donnell, ${ }^{8,12,13}$ Murray A Mittleman ${ }^{1,2}$

To cite: Dorans KS, Wilker EH, Li W, et al. Residential proximity to major roads, exposure to fine particulate matter and aortic calcium: the Framingham Heart Study, a cohort study. BMJ Open 2017;7:e013455. doi:10.1136/bmjopen-2016013455

- Prepublication history and additional material is available. To view please visit the journal (http://dx.doi.org/ 10.1136/bmjopen-2016013455).

Received 13 July 2016 Revised 28 October 2016 Accepted 22 December 2016

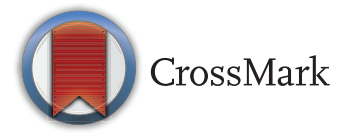

For numbered affiliations see end of article.

Correspondence to Murray A Mittleman; mmittlem@hsph.harvard.edu

\section{ABSTRACT}

Objectives: Traffic and ambient air pollution exposure are positively associated with cardiovascular disease, potentially through atherosclerosis promotion. Few studies have assessed associations of these exposures with thoracic aortic calcium Agatston score (TAC) or abdominal aortic calcium Agatston score (AAC), systemic atherosclerosis correlates. We assessed whether living close to a major road and residential fine particulate matter $\left(\mathrm{PM}_{2.5}\right)$ exposure were associated with TAC and AAC in a Northeastern US cohort.

Design: Cohort study.

Setting: Framingham Offspring and Third Generation participants residing in the Northeastern USA.

Participants and outcome measures: Among 3506 participants, mean age was 55.8 years; $50 \%$ female. TAC was measured from 2002 to 2005 and AAC up to two times (2002-2005; 2008-2011) among participants from the Framingham Offspring or Third Generation cohorts. We first assessed associations with detectable TAC (logistic regression) and AAC (generalised estimating equation regression, logit link). As aortic calcium scores were right skewed, we used linear regression models and mixed-effects models to assess associations with natural log-transformed TAC and $A A C$, respectively, among participants with detectable aortic calcium. We also assessed associations with AAC progression. Models were adjusted for demographic variables, socioeconomic position indicators and time.

Results: There were no consistent associations of major roadway proximity or $\mathrm{PM}_{2.5}$ with the presence or extent of TAC or AAC, or with AAC progression. Some estimates were in the opposite direction than expected.

Conclusions: In this cohort from a region with relatively low levels of and variation in $\mathrm{PM}_{2.5}$, there were no strong associations of proximity to a major road or $\mathrm{PM}_{2.5}$ with the presence or extent of aortic calcification, or with AAC progression.

\section{Strengths and limitations of this study}

- In this study, we used two measures of aortic calcification, abdominal aortic calcium score (AAC) and thoracic aortic calcium score (TAC), which are markers of systemic atherosclerosis.

- We assessed the associations of two different exposures, residential proximity to a major roadway and residential exposure to fine particulate matter $\left(\mathrm{PM}_{2.5}\right)$, with the presence and extent of TAC and AAC and with AAC progression.

- Many participants did not have detectable calcification and this study was carried out in the Northeastern USA, a region with relatively low levels of and variation in $\mathrm{PM}_{2.5}$, which might have limited our ability to detect associations with aortic calcification and may limit generalisability of the results.

- Because we did not randomly allocate where people live, there is the possibility for residual or unmeasured confounding, though we did adjust for many potential confounders.

- Exposure measurement error and the relatively short time period between AAC measurements (median 6.2 years) may have limited power to detect associations with AAC progression.

\section{INTRODUCTION}

Many studies have found that long-term particulate matter air pollution exposure is associated with cardiovascular disease (CVD) morbidity and mortality. ${ }^{1-5}$ Particulate matter air pollution may lead to cardiovascular disease by initiating or accelerating atherosclerosis. In susceptible animal models, exposure to particulate matter has been shown to lead to atherosclerosis progression. $^{6-8}$ Epidemiological studies have also suggested ambient air pollution and traffic 
are positively associated with markers of atherosclerosis. ${ }^{9-16}$ Possible mechanisms through which particulate matter could lead to atherosclerosis promotion include imbalance of the autonomic nervous system, a systemic oxidative stress and inflammatory response and, possibly, the transportation of particles directly into arterial blood circulation. ${ }^{17-19}$

Even in regions with relatively low levels of fine particulate matter (aerodynamic diameter $\leq 2.5 \mu \mathrm{m}, \mathrm{PM}_{2.5}$ ), studies have reported positive associations between $\mathrm{PM}_{2.5}$ and cardiovascular or all-cause mortality. ${ }^{20}{ }^{21}$ For instance, $\mathrm{PM}_{2.5}$ has been positively associated with mortality in New England..$^{22}$ In Massachusetts, traffic exposure has been linked to adverse cardiovascular outcomes ${ }^{23-25}$ and $\mathrm{PM}_{2.5}$ to acute myocardial infarction. ${ }^{26}$ Among elderly men living in the Boston area, carotid intima-media thickness (CIMT), a marker of subclinical atherosclerosis, was associated with black carbon, a traffic correlate. ${ }^{27}$

Though many studies have found positive associations between particulate matter air pollution and CIMT, ${ }^{14}{ }^{15}{ }^{27}$ only two have explored associations of these exposures with aortic calcification, ${ }^{13} 28$ a marker of systemic atherosclerosis and of a later stage of atherosclerotic disease than CIMT. Calcification in the thoracic aorta or abdominal aorta is correlated with other markers of subclinical atherosclerosis ${ }^{29-33}$ and predicts incident cardiovascular disease. ${ }^{34-41}$

In a study in Germany, $\mathrm{PM}_{2.5}$ and night-time traffic noise were associated with thoracic aortic calcium Agatston score (TAC).${ }^{13}$ Living closer to a highly trafficked road was associated with TAC, though this was attenuated after adjusting for night-time traffic noise. ${ }^{13}$ In a multicity US study, residential $\mathrm{PM}_{2.5}$ exposure was weakly associated with higher risk of detectable abdominal aortic calcium Agatston score $(\mathrm{AAC}) ;{ }^{28}$ there was no association with the quantity of AAC. ${ }^{28}$ There was no association of residential proximity to a major roadway with AAC.

We built on prior work and assessed associations of residential distance to a major roadway and residential exposure to spatially resolved average $\mathrm{PM}_{2.5}$ with TAC, AAC and AAC progression among Framingham Offspring or Third Generation cohort participants living in the Northeastern USA. TAC and AAC were measured from 2002 to 2005 and AAC was measured again from 2008 to 2011. During this period, $\mathrm{PM}_{2.5}$ levels in this Northeastern US study region were generally lower than levels in German-based ${ }^{13}$ and US-based ${ }^{28}$ studies that assessed associations with TAC and AAC. This work adds to studies that have assessed associations of particulate matter or traffic with other markers of atherosclerosis in Boston $^{27}$ and the Northeastern USA. ${ }^{42}$

\section{METHODS}

Study participants

The study population consists of participants from the Framingham Offspring and Third Generation cohorts, which have previously been described. ${ }^{43}{ }^{44}$ A subset of the participants underwent the first round of multidetector CT (MDCT) scans from 2002 to $2005 .{ }^{29} 45$ For this substudy, exclusion criteria included weighing $\geq 350$ pounds, age $<35$ years (men) or $<40$ years (women) and pregnancy. From 2008 to 2011, some participants scanned from 2002 to 2005 and additional Framingham Heart Study (FHS) participants underwent MDCT scans. Overall, 3530 participants (Offspring: 1419; Third Generation: 2111) had aortic calcium measured from 2002 to 2005 and 2749 participants (Offspring: 1320; Third Generation: 1429) had AAC measured from 2008 to 2011. Age of participants ranged from early adulthood to older age (many participants were middle aged), approximately half were women and participants were largely free of clinically apparent CVD. For these analyses, we included participants who had at least one aortic calcium score from the first or second MDCT period, lived in the Northeastern USA and were not missing exposure or covariate information. For scans carried out during the first MDCT round, we included participants who attended either Offspring Examination 7 (1998-2001) or Third Generation Examination 1 (2002-2005). For scans carried out during the second round, we included participants who attended either Offspring Examination 8 (2005-2008) or Third Generation Examination 2 (2008-2011). All participants provided written consent for FHS examinations and the MDCT scans.

\section{Participant characteristics}

At examination visits, each participant underwent a physical examination, physician interview and laboratory tests. Details of visits ${ }^{44}{ }^{46}$ and descriptions ${ }^{47}$ of blood pressure measurement, definitions of diabetes and use of antihypertensive and lipid-lowering medications have previously been described. Following published FHS criteria, ${ }^{44}{ }^{46}$ a panel of three investigators determined history of clinically apparent CVD (coronary heart disease, intermittent leg claudication, heart failure, stroke or transient ischaemic attack). Using each study participant's primary address, we used US Census 2000 data to assign the median value of owner-occupied housing units at the census-tract level, an area-level marker of socioeconomic position.

\section{Exposure assessment}

Distance to roadway: ArcGIS was used to geocode primary addresses of participants (Environmental Systems Research Institute (Esri), Redlands, CA). Using address reported at Offspring Examination 7 or Third Generation Examination 1, we evaluated distance to nearest major roadway, defined as A1, A2 or A3 road (US Census Features Class). Research has shown that particulate matter mass concentration and elemental carbon approach urban background levels within $~ 100$ to $400 \mathrm{~m}$ from major roadways. ${ }^{48}$ In an effort to reflect the decay of traffic-related pollution levels, we 
categorised distance to major roadway as $<50,50$ to $<200,200$ to $<400$ and 400 to $<1000 \mathrm{~m}$. We also assessed the association between the natural logarithm of distance to a major roadway and aortic calcium, based on our prior findings of log-linear associations between distance to a major roadway and cardiovascular health outcomes. $^{24} 47{ }^{49}$ For natural $\log$ of distance to nearest major roadway, we present results scaled to the IQR of distance to a major roadway, contrasting participants living $417.9 \mathrm{~m}$ to those living $58.0 \mathrm{~m}$ from the nearest major roadway. Participants residing $1000 \mathrm{~m}$ or further from a major roadway are likely to reside in rural areas, in which distance to a major road is unlikely to be an indicator of traffic-related pollution exposure. We therefore excluded observations from participants living $\geq 1000 \mathrm{~m}$ from a major road (572 observations, $11 \%$ ) in all analyses of distance to roadway.

Spatially resolved average $P M_{2.5}$ : We used estimates of daily $\mathrm{PM}_{2.5}$ levels at participant residential address, estimated by a spatiotemporal model to calculate each participant's average residential $\mathrm{PM}_{2.5}$ exposure. Kloog et $a \check{l}^{0}$ predicted daily $\mathrm{PM}_{2.5}$ at a scale of $1 \times 1 \mathrm{~km}$ across the Northeastern USA (New England, New York and New Jersey) using satellite-derived measurements of aerosol optical depth (AOD; a quantitative measure of particle abundance in the atmospheric column), $\mathrm{PM}_{2.5}$ measurements from monitoring stations and meteorological and land-use regression terms. There was excellent out-of-sample 10-fold cross-validated $\mathrm{R}^{2}$ (mean out of sample $\mathrm{R}^{2}=0.88$; year-to-year variation $0.82-0.90$ ) and a cross-validated slope of observed versus predicted $\mathrm{PM}_{2.5}$ concentrations of 0.99 (year-to-year variation $0.98-1.01$ ) among $1 \times 1 \mathrm{~km}$ grid cells that had a $\mathrm{PM}_{2.5}$ monitor measurement and AOD measurement. ${ }^{50}$ For each $\mathrm{PM}_{2.5}$ monitoring site, daily $\mathrm{PM}_{2.5}$ residuals (predicted vs measured) were regressed against local spatial and temporal variables. Using the fit of this model, we estimated daily predictions at home address for each residential address that represent deviations from grid predictions. To estimate total daily $\mathrm{PM}_{2.5}$, we summed the daily grid prediction and daily local residual $\mathrm{PM}_{2.5}$ prediction corresponding to each address. We then averaged these daily total $\mathrm{PM}_{2.5}$ predictions over the year to estimate annual average residential $\mathrm{PM}_{2.5}$. As has been previously done, ${ }^{47} 51-53$ we used the same index year for all observations. This approach aims to preserve $\mathrm{PM}_{2.5}$ spatial variation among residential locations, while minimising $\mathrm{PM}_{2.5}$ secular trend influences. For the main analyses, we chose the year 2003. To assess sensitivity of the results to choice of $\mathrm{PM}_{2.5}$ index year, we also ran analyses using $\mathrm{PM}_{2.5}$ averaged from 2003 to 2009 . We scaled results to the IQR of $2003 \mathrm{PM}_{2.5}\left(1.4 \mu \mathrm{g} / \mathrm{m}^{3}\right)$.

\section{Aortic artery calcium}

For the first round of scans, eight-slice MDCT technology was used to carry out scans of the chest and abdomen (LightSpeed Ultra, General Electric, Milwaukee, Wisconsin, USA).$^{29} 45$ For the second round, 64-slice MDCT scanners (General Electric Discovery VCT 64-slice PET/CT scanner, GE Healthcare) were used. For the chest, $482.5 \mathrm{~mm}$ slices were acquired from the carina to the diaphragm $(120 \mathrm{kVp}, 500 \mathrm{~ms}$ gantry rotation time, tube current $320 / 400 \mathrm{~mA} \quad(<100$ and $\geq 100 \mathrm{~kg}$ of body weight, respectively $\left.{ }^{29}\right)$. In the abdomen, using the top of the S1 vertebrae as the anatomic landmark of the lower field, slices were obtained for a total coverage of $15 \mathrm{~cm}$ in the Z-direction. ${ }^{29}$ Abdominal imaging parameters were: $120 \mathrm{kVp}, 400 \mathrm{~mA}$ and gantry rotation time of $500 \mathrm{~ms} .{ }^{29}$ A calcified lesion was defined as an area of $\geq 3$ connected pixels with an attenuation $>130$ Hounsfield units. ${ }^{45}$ A $\mathrm{A}$ modified Agatston score was calculated by multiplying the area of each lesion by a weighted attenuation score based on maximal lesion attenuation. TAC was measured twice in each participant (sequential scans); we use average TAC from these two scans.

\section{Statistical analyses}

Owing to the zero-inflated and heavily right-skewed distribution of TAC, we used a two-step approach to separately assess associations of exposure with detectable TAC and then with the amount of TAC among participants with TAC $>0$. For the binary outcome of detectable TAC, we used logistic regression. We used linear regression models to examine associations with the amount of TAC among participants with TAC $>0$. Owing to its skewed distribution, we modelled natural log-transformed TAC. We estimated 95\% CIs by bootstrapping 1000 samples and reported percentile CIs, as we did not expect residuals to be normally distributed. ${ }^{55}$ We used a similar approach for AAC. To account for repeated AAC measures among $51 \%$ of participants, we used generalised estimating equation logistic regression, robust SEs and an unstructured correlation structure for the binary outcome of detectable AAC. To assess associations with the amount of natural log-transformed AAC among participants with AAC $>0$, we used linear mixed-effects models (95\% CIs block bootstrapped).

We also assessed associations with AAC incidence and with annual change in AAC. We defined AAC incidence as progressing from $\mathrm{AAC}=0$ to detectable $\mathrm{AAC}(\mathrm{AAC}>0)$. Prior work in FHS has shown that CVD risk factors were associated with incident AAC. ${ }^{56}$ We used logistic regression to evaluate associations with odds of incident AAC. Next, we used a linear mixed-effects modelling approach that simultaneously assessed associations of exposure with a participant's first measured AAC and change in AAC per year (the latter assessed by including interaction terms of time between scans with exposure; block bootstrapped CIs). ${ }^{21} 5455$ We used all observations for this second approach, including participants scanned at one or both rounds of MDCT scans.

We adjusted for covariates considered a priori to be potential confounders of the air pollution and atherosclerosis relationship. In all models, we adjusted for age and age $^{2}$ at scan, sex, body mass index, smoking status 
(current, former, never), pack-years, individual-level educational attainment (high school or less, some college, college graduate), median census-tract value of owneroccupied housing units (quartiles), cohort (Offspring or Third Generation) and time.

We focused on parameter estimation and the overall pattern of results, rather than results of specific hypothesis tests, and so do not report $\mathrm{p}$ values. We present all point estimates with 95\% CIs. We used SAS V.9.3 (SAS Institute, Cary, North Carolina, USA) and Stata V.12 (StataCorp, College Station, Texas, USA) for analyses.

\section{Sensitivity analyses}

To assess robustness of our findings, we ran sensitivity analyses. We assessed whether results were similar when we only adjusted for age and sex. We also assessed whether results changed when we added other potential confounders. First, we additionally adjusted for potential confounders or predictors of aortic calcium: physical activity (tertiles of physical activity index ${ }^{57}$ ), alcohol $(0,0$ to 7 and $\geq 7$ drinks per week) and menopausal status in women (periods stopped for 1 year or more before examination). We then further adjusted for variables that could be confounders, outcome predictors or mediators: diabetes, systolic blood pressure, diastolic blood pressure, total cholesterol/high-density lipoprotein (HDL) ratio, triglycerides, antihypertensive medication and lipid-lowering medication.

We tested for heterogeneity of associations between distance to major roadway and $\mathrm{PM}_{2.5}$ with the presence or extent of aortic calcium by: sex, age $(\leq 65,>65)$, cohort (Offspring, Third Generation) and American College of Cardiology/American Heart Association's 10-year predicted risk of atherosclerotic CVD (tertiles: $0.1-1.6 \%, 1.6-5.3 \%, 5.3-81.3 \%) .{ }^{58}$ To run these analyses, we included interaction terms between the exposure and covariate of interest. Owing to sparse data, we did not assess for heterogeneity by 10-year atherosclerotic CVD risk for the outcomes of detectable TAC or $\log _{e}$ (TAC) or by age for the outcome of detectable AAC. We also assessed whether results differed when we restricted to participants without clinically apparent CVD (4891 observations, $92 \%$ ).

We assessed associations of aortic calcium with residential distance to nearest A1 or A2 road (rather than nearest A1, A2 or A3 road), as A1 and A2 roads are larger than A3 roads. In an effort to take into account long-term secular $\mathrm{PM}_{2.5}$ trends, we adjusted for MDCT scan year as a categorical variable. We also ran AAC analyses separately for the first and second rounds of MDCT scans, to assess whether results differed by period.

We used restricted cubic splines to assess linearity of the exposure-outcome relationships for natural log distance to roadway and $\mathrm{PM}_{2.5}{ }^{59}$ and created plots using the POSTRCSPLINE package in Stata. ${ }^{60}$

To assess the robustness of the generalised estimating equation regression results, we reran the binary AAC $>0$ analyses using mixed-effects logistic regression. In line with prior work, ${ }^{13}$ we used linear regression to assess associations with $\log _{\mathrm{e}}(\mathrm{TAC}+1)$ and mixed-effects linear regression to assess associations with $\log _{\mathrm{e}}(\mathrm{AAC}+1)$. As in prior FHS analyses ${ }^{61}$ we also assessed associations with TAC and AAC greater than the 75th or 90th age-specific and sex-specific centiles among a healthy referent subpopulation, using logistic regression for TAC and generalised estimating equation regression (logit link, unstructured covariance and robust SEs) for AAC.

\section{RESULTS}

\section{Study participants}

Participant characteristics are listed in table 1 and online supplementary table S1. Of the 6279 aortic calcium scores measured during either the first or second MDCT round, these analyses included 5328 observations from 3506 participants. Mean age of participants during the first MDCT round was 52.6 years, and for the second round was 59.6 years. In total, women contributed $50 \%$ of observations. In the second round of MDCT scans, participants were more likely to report being on hypertension medication $(37 \%$ vs $20 \%)$ or lipid medication ( $40 \%$ vs $15 \%$ ) and were less likely to be current smokers $(8 \%$ vs $13 \%)$ than participants from the first round of MDCT scans.

\section{Exposure distributions}

Exposure distributions are summarised in table 2. Twenty-three per cent of observations came from participants who lived within $50 \mathrm{~m}$ of a major roadway; the median distance to the closest major roadway was $201 \mathrm{~m}$. In 2003, median residential $\mathrm{PM}_{2.5}$ concentration was $10.7 \mu \mathrm{g} / \mathrm{m}^{3}$. The current annual $\mathrm{PM}_{2.5}$ US Environmental Protection Agency (EPA) annual National Air Quality Standard (NAAQS) is $12 \mu \mathrm{g} / \mathrm{m}^{3}$.

\section{Associations with odds of detectable aortic calcium and continuous aortic calcium}

There were no consistent associations of residential proximity to a major roadway or $\mathrm{PM}_{2.5}$ with the presence or extent of TAC or AAC (table 3). Some of the associations were in the opposite direction than expected.

\section{Associations with change in AAC}

There were 1811 participants with AAC measured during MDCT round one and round two; 818 participants had no detectable AAC during MDCT round one. Of these, $30 \%$ had detectable AAC during round two. We found no association of residential distance to major roadway or $\mathrm{PM}_{2.5}$ with incident AAC (table 4, figure 1). Distance to nearest major road and $\mathrm{PM}_{2.5}$ were also not associated with annual change in AAC (table 4, figure 1).

\section{Sensitivity analyses}

We did not find evidence of non-linearity of the associations between any of the exposures with the presence or extent of aortic calcium, or with the incidence of AAC. 
Table 1 Characteristics of study participants

\begin{tabular}{|c|c|c|}
\hline \multirow[b]{2}{*}{ Characteristics } & \multicolumn{2}{|l|}{ mean $\pm S D$ or $n(\%)$} \\
\hline & CT 2002-2005 (N=2945) & CT 2008-2011 ( $\mathrm{N}=2383)$ \\
\hline Age at CT scan (years) & $52.6 \pm 11.8$ & $59.6 \pm 12.0$ \\
\hline Male sex, \% & $1506(51)$ & $1165(49)$ \\
\hline Offspring, \% & $1201(41)$ & $1155(48)$ \\
\hline \multicolumn{3}{|l|}{ Education (years) } \\
\hline Some college & $954(32)$ & $771(32)$ \\
\hline College graduate & $1301(44)$ & $1063(45)$ \\
\hline Median census value owner-occupied housing, \$ & $221976 \pm 100340$ & $222381 \pm 102327$ \\
\hline Current smokers, \% & $381(13)$ & $179(8)$ \\
\hline Former smokers, \% & $1109(38)$ & $1027(43)$ \\
\hline \multicolumn{3}{|l|}{ Pack-years } \\
\hline Current smokers & $30.5 \pm 14.4$ & $33.5 \pm 15.7$ \\
\hline Former smokers & $17.5 \pm 17.9$ & $17.5 \pm 17.1$ \\
\hline Alcohol (average drinks per week) & $4.9 \pm 7.3$ & $4.6 \pm 7.3$ \\
\hline Physical Activity Index & $37.6 \pm 7.4$ & $36.1 \pm 6.3$ \\
\hline Menstrual periods stopped ${ }^{\star}, \%$ & $761(53)$ & $936(77)$ \\
\hline Diabetes history, \% & $168(6)$ & $223(9)$ \\
\hline Clinically apparent CVD at CT scan, \% & $216(7)$ & $221(9)$ \\
\hline Body mass index $\left(\mathrm{kg} / \mathrm{m}^{2}\right)$ & $27.8 \pm 5.3$ & $28.5 \pm 5.3$ \\
\hline Hypertension medications, \% & $590(20)$ & $891(37)$ \\
\hline Systolic blood pressure (mm Hg) & $122 \pm 16$ & $123 \pm 16$ \\
\hline Diastolic blood pressure (mm $\mathrm{Hg})$ & $76 \pm 9$ & $74 \pm 9$ \\
\hline Lipid medications, \% & $438(15)$ & $957(40)$ \\
\hline Triglycerides (mg/dL) & $129 \pm 92$ & $118 \pm 79$ \\
\hline Total cholesterol/HDL & $4.0 \pm 1.3$ & $3.5 \pm 1.1$ \\
\hline 10-year predicted risk of CVD†, \% & $0.05 \pm 0.07$ & $0.07 \pm 0.09$ \\
\hline \multicolumn{3}{|l|}{ MDCT scan results } \\
\hline $\mathrm{TAC}>0, \%$ & $716(25)$ & $\mathrm{N} / \mathrm{A}$ \\
\hline TAC, among those with TAC>0‡ & $156.6(712.8)$ & N/A \\
\hline$A A C>0, \%$ & $1608(55)$ & $1604(67)$ \\
\hline AAC, among those with $\mathrm{AAC}>0 \ddagger$ & $464.6(2066.8)$ & $745.5(3107.0)$ \\
\hline \multicolumn{3}{|c|}{$\begin{array}{l}{ }^{*} \text { Among women. } \\
\text { †American College of Cardiology/American Heart Association } 2013 \text { 10-year predicted risk of atherosclerotic CVD; Median, IQR. }{ }^{58} \\
\text { †Median, IQR. } \\
\text { Data calculated from } 5328 \text { observations, from } 3506 \text { participants with a TAC measurement or at least one AAC measurement. Number of } \\
\text { missing observations: TAC: 45; AAC: } 16 \text {; alcohol: 4; physical activity index: 47; diastolic blood pressure: 4; triglycerides: 10; total cholesterol/ } \\
\text { HDL: 15; 10-year predicted CVD risk: } 1223 \text {. } \\
\text { AAC, abdominal aortic calcium Agatston score; CVD, cardiovascular disease; HDL, high-density lipoprotein; MDCT, multidetector CT; TAC, } \\
\text { thoracic aortic calcium Agatston score. }\end{array}$} \\
\hline
\end{tabular}

Table 2 Distributions of distance to roadway, $\mathrm{PM}_{2.5}$

\begin{tabular}{|c|c|c|c|}
\hline Exposure & Median (IQR) or $\mathbf{n}(\%)$ & Range (min, max) & Range (5th-95th) \\
\hline Proximity to major roadway $(m)^{*}$ & $201(360)$ & $0.01-999.7$ & $6.9-816.1$ \\
\hline Total $\mathrm{PM}_{2.5}\left(\mu \mathrm{g} / \mathrm{m}^{3}\right), 2003 \dagger$ & $10.7(1.4)$ & $2.9-26.7$ & $8.2-12.6$ \\
\hline Total $\mathrm{PM}_{2.5}\left(\mu \mathrm{g} / \mathrm{m}^{3}\right)$, 2003-2009† & $9.8(1.1)$ & $2.6-17.2$ & $7.3-11.1$ \\
\hline \multicolumn{4}{|l|}{ Residential proximity in categories } \\
\hline$<50 \mathrm{~m}$ & $1104(23 \%)$ & & \\
\hline 50 to $<200 \mathrm{~m}$ & $1265(27 \%)$ & & \\
\hline 200 to $<400 \mathrm{~m}$ & $1114(23 \%)$ & & \\
\hline 400 to $<1000 m$ & $1273(27 \%)$ & & \\
\hline
\end{tabular}


Table 3 Associations of distance to a major roadway and $\mathrm{PM}_{2.5}$ with $\mathrm{TAC}$ and $\mathrm{AAC}$ *

\begin{tabular}{|c|c|c|c|}
\hline & \multicolumn{3}{|l|}{ TAC† } \\
\hline & \multirow{2}{*}{$\begin{array}{l}\text { TAC>0 } \\
\text { OR } 95 \% \mathrm{CI}\end{array}$} & \multicolumn{2}{|c|}{ Linear regression (among those with $\mathrm{TAC}>0$ ) } \\
\hline & & Per cent difference & $95 \% \mathrm{Cl}$ \\
\hline$<50 \mathrm{~m}$ & 0.85 (0.59 to 1.22$)$ & -0.6 & $(-30.9$ to 52.0$)$ \\
\hline 50 to $<200 \mathrm{~m}$ & $0.70(0.49$ to 1.00$)$ & -0.4 & $(-35.4$ to 51.0$)$ \\
\hline 200 to $<400 \mathrm{~m}$ & 0.91 (0.64 to 1.29$)$ & -16.1 & $(-43.5$ to 28.7$)$ \\
\hline \multicolumn{4}{|l|}{400 to $<1000 \mathrm{~m}$} \\
\hline Log of distance to major road $\ddagger$ & $1.06(0.90$ to 1.24$)$ & -2.6 & $(-19.1$ to 14.4$)$ \\
\hline $2003 \mathrm{PM}_{2.5}\left(\mu \mathrm{g} / \mathrm{m}^{3}\right) \S$ & 0.89 (0.78 to 1.02$)$ & 2.4 & $(-13.0$ to 20.8$)$ \\
\hline \multirow[t]{4}{*}{ 2003-2009 $\mathrm{PM}_{2.5}\left(\mu \mathrm{g} / \mathrm{m}^{3}\right) \S$} & $0.88(0.76$ to 1.01$)$ & 19.6 & $(-1.4$ to 45.9$)$ \\
\hline & \multicolumn{3}{|l|}{ AACI } \\
\hline & \multirow{2}{*}{$\begin{array}{l}\text { AAC }>0 \\
\text { OR } 95 \%\end{array}$} & \multicolumn{2}{|c|}{ Linear mixed effects (among those with AAC>0) } \\
\hline & & Per cent difference & $95 \% \mathrm{Cl}$ \\
\hline$<50 \mathrm{~m}$ & $0.85(0.65$ to 1.11$)$ & 3.2 & $(-12.9$ to 22.8$)$ \\
\hline 50 to $<200 \mathrm{~m}$ & 0.79 (0.62 to 1.00$)$ & -11.8 & $(-23.1$ to 5.8$)$ \\
\hline 200 to $<400 \mathrm{~m}$ & $0.96(0.75$ to 1.23$)$ & -3.8 & $(-17.4$ to 13.5$)$ \\
\hline 400 to $<1000 \mathrm{~m}$ & 1.00 & & \\
\hline Log of distance to major road $\ddagger$ & 1.09 (0.96 to 1.23$)$ & -0.4 & $(-8.6$ to 7.9$)$ \\
\hline $2003 \mathrm{PM}_{2.5}\left(\mu \mathrm{g} / \mathrm{m}^{3}\right) \S$ & 0.99 (0.90 to 1.08$)$ & 2.2 & $(-3.2$ to 9.9$)$ \\
\hline 2003-2009 $\mathrm{PM}_{2.5}\left(\mu \mathrm{g} / \mathrm{m}^{3}\right) \S$ & $0.97(0.89$ to 1.07$)$ & 5.8 & $(-0.1$ to 14.5$)$ \\
\hline \multicolumn{4}{|c|}{ 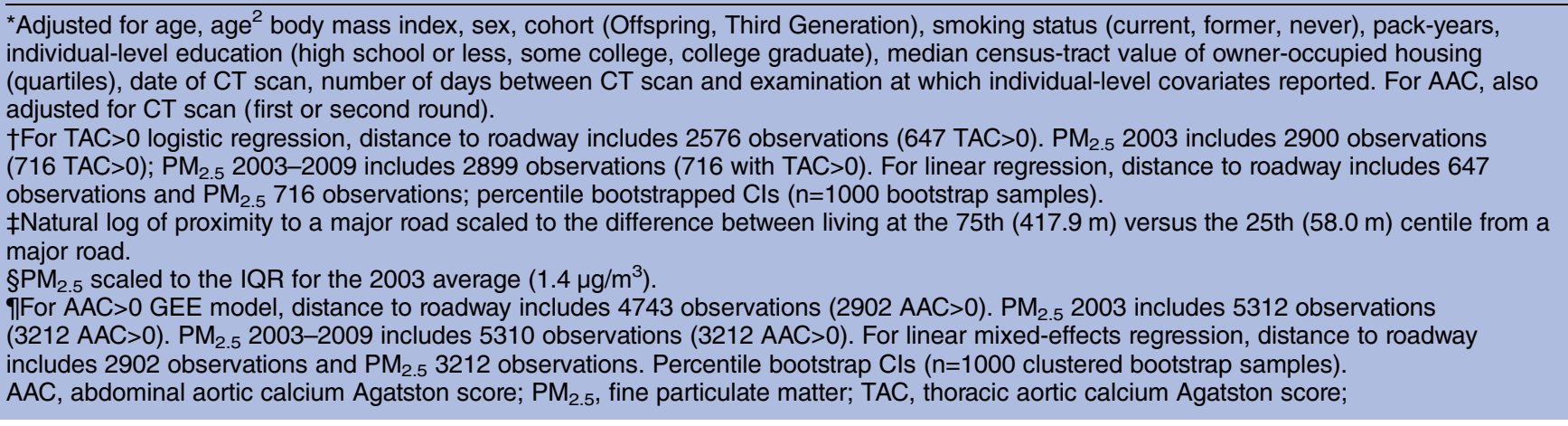 } \\
\hline
\end{tabular}

When we only adjusted for age and sex, results were not changed substantially. We observed similar results when we also adjusted for physical activity, alcohol, menopausal status, diabetes, systolic blood pressure, diastolic blood pressure, total cholesterol/HDL ratio, triglycerides, antihypertensive medication and lipid-lowering medication or when we adjusted for year of CT scan as categorical variable.

We did not observe consistent patterns of heterogeneity of associations with the presence or extent of aortic calcium by age, sex (men vs women), cohort or predicted 10-year risk of atherosclerotic CVD (see online supplemental table S2). Of note, there was sparse data in some subgroups. When we restricted to participants without clinically apparent CVD (92\%), results were similar.

When we used a different $\mathrm{PM}_{2.5}$ averaging period (2003-2009 instead of 2003), some point estimates were stronger, though CIs were wide (tables 3 and 4). We did not observe consistent associations of distance to nearest A1 or A2 road with the presence or extent of aortic calcium or with the incidence of AAC, and some of the associations were in the opposite direction than expected. Results were similar when we ran the AAC analyses separately by MDCT round.

When we ran the AAC $>0$ analysis with a mixed logistic regression, instead of the generalised estimating equation regression, we obtained similar results, though with wider CIs. Distance to a major roadway and $\mathrm{PM}_{2.5}$ were not strongly associated with $\log _{\mathrm{e}}(\mathrm{TAC}+1)$ or with $\mathrm{TAC}$ greater than the 75th or 90th age-specific and sexspecific healthy referent cut points. Similarly, we did not see associations with $\log _{\mathrm{e}}(\mathrm{AAC}+1)$ or with $\mathrm{AAC}$ greater than the 75th or 90th age-specific and sex-specific healthy cut points.

\section{DISCUSSION}

We assessed two residential exposures: distance to a major roadway, which is a surrogate of local traffic exposure, and $\mathrm{PM}_{2.5}$, which is emitted by a variety of local and regional pollution sources, including traffic. We found no consistent or strong associations between these exposures and the presence or extent TAC or AAC or with AAC progression. These findings were robust across a range of sensitivity analyses. 
Table 4 Associations of distance to a major roadway, $\mathrm{PM}_{2.5}$ with incidence of newly detectable $\mathrm{AAC}$ and change in $\mathrm{AAC}$

\begin{tabular}{llll}
\hline & Incident AAC* & \multicolumn{2}{l}{ Annual change in AAC† } \\
\cline { 2 - 4 } & OR 95\% Cl & Change & 95\% Cl \\
\hline$<50 \mathrm{~m}$ & $0.74(0.45$ to 1.23$)$ & 23.4 & $(-6.7$ to 55.9$)$ \\
50 to $<200 \mathrm{~m}$ & $0.66(0.41$ to 1.07$)$ & -28.9 & $(-59.4$ to -3.1$)$ \\
200 to $<400 \mathrm{~m}$ & $0.74(0.44$ to 1.23$)$ & -9.1 & $(-31.7$ to 17.4$)$ \\
400 to $<1000 \mathrm{~m}$ & 1.00 & & \\
Log of distance to a major road $\neq$ & $1.15(0.91$ to 1.46$)$ & -5.8 & $(-21.7$ to 7.4$)$ \\
$2003 \mathrm{PM}_{2.5}\left(\mu \mathrm{gg} / \mathrm{m}^{3}\right) \S$ & $0.93(0.79$ to 1.10$)$ & 1.1 & $(-6.7$ to 9.7$)$ \\
$2003-2009 \mathrm{PM}_{2.5}\left(\mu \mathrm{g} / \mathrm{m}^{3}\right) \S$ & $0.95(0.79$ to 1.14$)$ & 7.5 & $(-2.6$ to 16.3$)$
\end{tabular}

${ }^{*}$ Adjusted for age at MDCT1 (age, age ${ }^{2}$ ) and covariates reported at Offspring Examination 7 or Generation 3 Examination 1: body mass index, sex, cohort, smoking status (current, former, never), pack-years, individual-level education (high school or less, some college, college graduate), median census-tract value of owner-occupied housing (quartiles), date of 1st scan, number of days between scan and examination at which individual-level covariates reported, time between MDCT1 and MDCT2.

†Adjusted for age at 1st scan (age, age ${ }^{2}$, body mass index, sex, cohort, scan (first or second round), smoking status (current, former, never), pack-years, individual-level education (high school or less, some college, college graduate), median census-tract value of owner-occupied housing (quartiles), date of scan, number of days between scan and examination at which individual-level covariates reported, time since 1st scan. Includes the following interaction terms with time since first scan: age at first scan (age, age ${ }^{2}$, sex, cohort. Percentile bootstrap Cls ( $n=1000$ clustered bootstrap samples).

$\ddagger$ Natural log of proximity to a major road scaled to the difference between living at 75 th $(417.9 \mathrm{~m})$ versus 25 th $(58.0 \mathrm{~m})$ centile from a major road. For AAC incidence, 716 observations ( 215 with incident AAC). For change in AAC mixed model, all 4743 observations are included (1625 with two AAC measurements).

\$PM $_{2.5}$ scaled to the IQR for the 2003 average $\left(1.4 \mu \mathrm{g} / \mathrm{m}^{3}\right)$. For $2003 \mathrm{PM}_{2.5}$ AAC incidence, 818 observations (248 with incident AAC). For 2003-2009 $\mathrm{PM}_{2.5}$ AAC incidence, 817 observations (248 with incident AAC). For $2003 \mathrm{PM}_{2.5}$ change in AAC mixed model, all 5312 observations are included (1811 with two AAC measurements). For 2003-2009 PM $_{2.5}$ change in AAC mixed model, all 5310 observations are included (1810 with two AAC measurements).

$\mathrm{AAC}$, abdominal aortic calcium Agatston score; $\mathrm{PM}_{2.5}$, fine particulate matter.

This study has several limitations. Though there is potential for residual or unmeasured confounding, we adjusted for many potential confounders, including individual and area-level markers of socioeconomic position. TAC and AAC are measured with error and are correlates, not direct estimates of systemic atherosclerosis. However, TAC and AAC predict incident $\mathrm{CVD}^{34-39}$ and are correlated with other markers of atherosclerosis. ${ }^{29-33}$ We previously reported no strong associations of these exposures with coronary artery calcium Agatston scores (CAC), another marker of atherosclerosis, in FHS. $^{42}$ However, our study would have been stronger if we had assessed associations with other atherosclerosis markers, such as CIMT. We did not assess associations with other markers of traffic, such as nitrogen dioxide. We do not have information on time activity patterns or time spent at home and our exposures are measured with error. However, we aimed to use residential location to estimate order of exposure levels. Importantly, we do not expect exposure measurement error to be related to TAC or AAC. We did not model year-to-year variability in exposures. However, results were similar when we adjusted for year of CT scan as a categorical variable in an effort to adjust for long-term time trends. Additionally, results were generally robust to choice of $\mathrm{PM}_{2.5}$ index period. We did not differentiate between type of major roadway (A1, A2, and A3); however, results were generally similar when we restricted to distance to A1 or A2 roadway only. Finally, the study population is predominantly of European ancestry and resides in the Northeastern USA. Though the study population included older adults, many participants were middle aged or younger. Results might not be generalisable to other populations.

There are also several strengths of this study. Unlike prior studies, we assess two measures of aortic calcification-in the thoracic and abdominal aorta-in the same study population. We assessed associations with incident AAC and annual change in AAC. We used a validated, spatially and temporally resolved $\mathrm{PM}_{2.5}$ model based on satellite and land use data to estimate individual exposure, rather than relying on community monitors. Additionally, we explored different exposures: distance to major roadway as a correlate of local traffic, and $\mathrm{PM}_{2.5}$, which has local and regional sources.

The two prior studies that assessed associations of distance to a major roadway or $\mathrm{PM}_{2.5}$ with aortic calcium score supported the presence of cross-sectional associations between proximity to major roadway and TAC and between $\mathrm{PM}_{2.5}$ and TAC and AAC. ${ }^{13}{ }^{28}$ In the Heinz Nixdorf Recall Study, carried out in an industrial region of Germany, residential $\mathrm{PM}_{2.5}$ was associated with the extent of TAC, even after adjusting for night-time traffic noise. ${ }^{13}$ Living close to a highly trafficked road was also associated with TAC, though this was attenuated when adjusted for night-time noise. ${ }^{13}$ In the Multiethnic Study of Atherosclerosis (MESA) study, based in the USA, higher residential $\mathrm{PM}_{2.5}$ exposure was weakly associated with a higher risk of detectable AAC. ${ }^{28}$ Associations were slightly stronger among participants whom the authors expected to have less exposure measurement errorthose with long-term residence near a $\mathrm{PM}_{2.5}$ monitor and those who did not recently work outside of the home. There were no associations of $\mathrm{PM}_{2.5}$ with the 


\section{A Associations with Newly Detectable AAC}

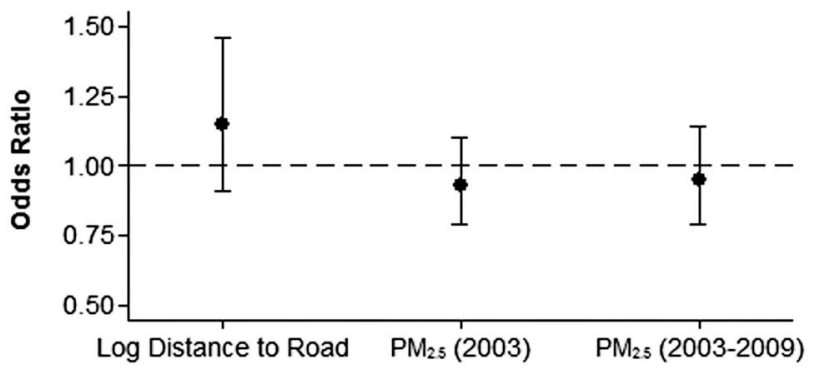

B

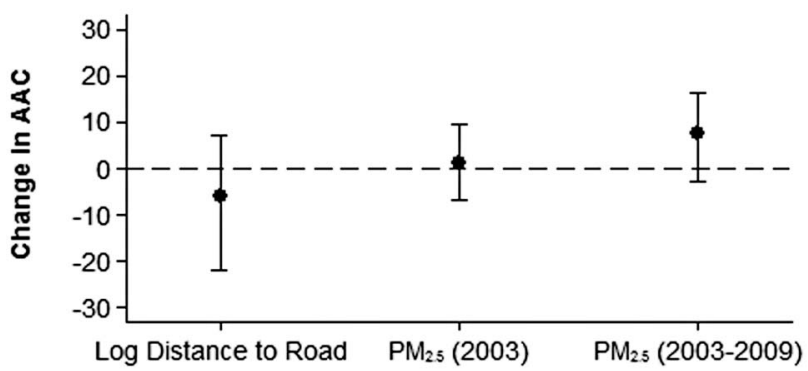

Figure 1 Associations of distance to a major roadway, $\mathrm{PM}_{2.5}$ with incidence of newly detectable AAC and change in AAC.

(A) adjusted for age at MDCT1 (age, age ${ }^{2}$ ) and covariates reported at Offspring Examination 7 or Generation 3

Examination 1: body mass index, sex, cohort, smoking status (current, former, never), pack-years, individual-level education (high school or less, some college, college graduate), median census-tract value of owner-occupied housing (quartiles), date of 1st scan, number of days between scan and examination at which individual-level covariates reported, time between MDCT1 and MDCT2. (B) Adjusted for age at 1st scan (age, $\mathrm{age}^{2}$, body mass index, sex, cohort, scan (first or second round), smoking status (current, former, never), pack-years, individual-level education (high school or less, some college, college graduate), median census-tract value of owner-occupied housing (quartiles), date of scan, number of days between scan and examination at which individual-level covariates reported, time since 1 st scan. Includes the following interaction terms with time since first scan: age at first scan (age, age ${ }^{2}$, sex, cohort. Percentile bootstrap Cls ( $n=1000$ clustered bootstrap samples). Natural log of proximity to a major road scaled to the difference between living at 75th $(417.9 \mathrm{~m})$ versus 25 th $(58.0 \mathrm{~m})$ centile from a major road. For AAC incidence, 716 observations ( 215 with incident AAC). For change in AAC mixed model, all 4743 observations are included (1625 with two AAC measurements). $\mathrm{PM}_{2.5}$ scaled to the IQR for the 2003 average $\left(1.4 \mu \mathrm{g} / \mathrm{m}^{3}\right)$. For $2003 \mathrm{PM}_{2.5}$ AAC incidence, 818 observations (248 with incident AAC). For 2003-2009 $\mathrm{PM}_{2.5}$ AAC incidence, 817 observations (248 with incident AAC). For $2003 \mathrm{PM}_{2.5}$ change in AAC mixed model, all 5312 observations are included (1811 with two AAC measurements). For 2003-2009 $\mathrm{PM}_{2.5}$ change in AAC mixed model, all 5310 observations are included (1810 with two AAC measurements). AAC, abdominal aortic calcium Agatston score; MDCT, multidetector $\mathrm{CT}$; $\mathrm{PM}_{2.5}$, fine particulate matter. extent of AAC or of distance to a major roadway with the presence or extent of AAC.

Unlike prior studies, we assessed associations of these exposures with TAC and AAC in the same study region and population. We were also able to assess associations with AAC progression. We did not observe associations of these outcomes with residential distance to a major roadway or residential estimates of $\mathrm{PM}_{2.5}$. In analyses of associations with the presence of detectable TAC and AAC, we observed some estimates that were in the opposite direction than expected. For instance, higher $\mathrm{PM}_{2.5}$ exposure was associated with lower odds of detectable TAC, though CIs were wide. There is no clear biological rationale for this direction of association, as we would expect that higher $\mathrm{PM}_{2.5}$ would be associated with higher odds of detectable TAC. Though we adjusted for many potential confounders, these results could possibly be due to chance or residual confounding. The lack of association with AAC progression may have been due to the relatively short time period between AAC scans (median 6.2 years).

Importantly, there was little variation in average $\mathrm{PM}_{2.5}$ levels in our study, which reduced power to observe associations between $\mathrm{PM}_{2.5}$ and aortic calcification. Additionally, $\mathrm{PM}_{2.5}$ levels were lower in our study compared with the two existing studies that have studied air pollution and TAC or AAC. For instance, in our cohort, 2003 annual average $\mathrm{PM}_{2.5}$ predicted at residential address had a median of $10.7 \mu \mathrm{g} / \mathrm{m}^{3}$, while median 365-day $\mathrm{PM}_{2.5}$ average was $16.6 \mu \mathrm{g} / \mathrm{m}^{3}$ in the Heinz Nixdorf Recall Study. ${ }^{13}$ Additionally, use of diesel is much more common in Germany than in the USA and diesel exhaust may be more harmful than gasoline exhaust. Unlike our study, the Heinz Nixdorf Recall Study found an association between $\mathrm{PM}_{2.5}$ and the extent of TAC. ${ }^{13}$ As in MESA, we did not observe a consistent association between distance to a major roadway or $\mathrm{PM}_{2.5}$ with the extent of AAC. ${ }^{28}$ However, in contrast to our study, Allen and colleagues found a suggested weak positive association of $\mathrm{PM}_{2.5}$ with detectable AAC among MESA participants. ${ }^{28}$ In the MESA study region, mean 2000-2002 average $\mathrm{PM}_{2.5}$ ranged from $10.9 \mu \mathrm{g} / \mathrm{m}^{3}$ (St. Paul) to $22.8 \mu \mathrm{g} / \mathrm{m}^{3}$ (Los Angeles).

Studies have also assessed associations of traffic and particulate matter air pollution with CAC, which provides an estimate of total coronary atheroma ${ }^{62}$ and predicts coronary heart disease. ${ }^{63}$ We did not find evidence of strong associations of residential proximity to a major road or $\mathrm{PM}_{2.5}$ with the presence, extent or progression of CAC. ${ }^{42}$ In the Heinz Nixdorf Recall Study, residing closer to a major road (mean daily vehicle counts of 10 000-130 000) was associated with CAC; $\mathrm{PM}_{2.5}$ was only associated among study participants who had not recently been employed full time. ${ }^{9}$ In line with our findings in FHS, ${ }^{42}$ in cross-sectional analyses, PM $_{2.5}$ was not 
consistently associated with $\mathrm{CAC}$ in $\mathrm{MESA},{ }^{10}$ though there was an association of $\mathrm{PM}_{2.5}$ and nitrogen oxides with CAC progression in MESA. ${ }^{16}$

Importantly, the lack of associations of these exposures with artery calcification in our study population does not mean that ambient air pollution does not cause atherosclerosis. When considering policy implications, these results should be interpreted within the broader context of air pollution and cardiovascular disease literature. Studies in susceptible animals have found particulate matter exposure can lead to atherosclerosis progression. ${ }^{6-8}$ Additionally, controlled human and animal studies have found evidence that short-term ambient air pollution exposure can lead to proinflammatory and oxidative stress responses and autonomic nervous system imbalance, which could potentially lead to atherosclerosis. ${ }^{17-19}$ Age is strongly related to the presence and extent of detectable artery calcification. Though participant ages ranged from early to late adulthood, many were middle aged or younger and had little or no detectable calcification, thereby reducing power to detect associations in this study. As noted above, the Heinz Nixdorf Recall Study and MESA found evidence of positive associations of these exposures with artery calcification. Additionally, many studies have reported positive associations between particulate matter air pollution and CIMT. ${ }^{14}{ }^{15} 27$ Importantly, CIMT represents an earlier stage in vascular injury ${ }^{64}$ than artery calcification, which only directly measures calcified elements. Perhaps relatively recent particulate matter pollution exposure contributes more to the development of soft plaque than to progression to arterial calcification, which may be more influenced by longer term exposures. Potential future work could explore whether, in this population, $\mathrm{PM}_{2.5}$ and residential proximity to roadway are associated with measures of soft plaques.

In summary, in a cohort residing in a region with relatively low levels of and variation in $\mathrm{PM}_{2.5}$, we observed no strong associations between residential distance to a major roadway or $\mathrm{PM}_{2.5}$ with the presence, extent or progression of aortic calcification. These findings add to the prior studies exploring the association of traffic and $\mathrm{PM}_{2.5}$ with aortic calcification in humans.

\author{
Author affiliations \\ ${ }^{1}$ Department of Epidemiology, Harvard T.H. Chan School of Public Health, \\ Boston, Massachusetts, USA \\ ${ }^{2}$ Cardiovascular Epidemiology Research Unit, Department of Cardiology, Beth \\ Israel Deaconess Medical Center, Harvard Medical School, Boston, \\ Massachusetts, USA \\ ${ }^{3}$ Department of Environmental Health, Harvard T.H. Chan School of Public \\ Health, Boston, Massachusetts, USA \\ ${ }^{4}$ Division of Pulmonary, Critical Care and Sleep Medicine, Beth Israel \\ Deaconess Medical Center, Harvard Medical School, Boston, Massachusetts, \\ USA \\ ${ }^{5}$ Unit of Environmental Epidemiology, Institute of Environmental Medicine, \\ Karolinska Institutet, Stockholm, Sweden \\ ${ }^{6}$ Department of Biostatistics, Harvard T.H. Chan School of Public Health, \\ Boston, Massachusetts, USA
}

${ }^{7}$ Department of Geography and Environmental Development, Ben-Gurion University of the Negev, Beer Sheva, Israel

${ }^{8}$ National Heart, Lung, and Blood Institute's and Boston University's

Framingham Heart Study, Framingham, Massachusetts, USA

${ }^{9}$ Department of Mathematics and Statistics, Boston University, Boston, Massachusetts, USA

${ }^{10}$ Department of Biostatistics, Boston University School of Public Health, Boston, Massachusetts, USA

${ }^{11}$ Cardiac MR PET CT Program, Department of Radiology, Massachusetts General Hospital, Harvard Medical School, Boston, Massachusetts, USA

${ }^{12}$ Cardiology Section, Department of Medicine, Boston Veteran's Administration Health System, Boston, Massachusetts, USA

${ }^{13}$ Cardiovascular Medicine, Brigham and Women's Hospital, Harvard Medical School, Boston, Massachusetts, USA

Acknowledgements We thank the Framingham Offspring and Third Generation Study participants. We also thank the editors at BMJ Open and the reviewers, Dr Mark Miller and Dr Jess Lambrechtsen, for their thoughtful feedback.

Contributors All authors have contributed to the conception or design of the study and acquisition of the data. KSD analysed the data and EHW and WL reviewed the analysis. KSD wrote the first draft of the manuscript and all authors critically revised the manuscript. All authors have approved the manuscript.

Funding This work was supported by the National Heart, Lung, and Blood Institute (grant number T32HL007575) and the National Institute of Environmental Health Sciences (grant numbers R00 ES022243, F32ES023352) of the National Institutes of Health. This publication was made possible by US Environmental Protection Agency (USEPA) grant number RD-83479801. From the Framingham Heart Study of the National Heart, Lung, and Blood Institute of the National Institutes of Health and Boston University School of Medicine; this work was supported by the National Heart, Lung, and Blood Institute's Framingham Heart Study (contract numbers N01-HC-25195 and HHSN268201500001I).

Disclaimer The contents of this study are solely the responsibility of the grantee and do not necessarily represent the official views of the USEPA. Further, USEPA does not endorse the purchase of any commercial products or services mentioned in the publication. The views expressed in this manuscript are those of the authors and do not necessarily represent the views of the National Heart, Lung, and Blood Institute; the National Institutes of Health or the US Department of Health and Human Services

Competing interests KSD reports grants from National Institutes of Health during the conduct of this study and outside the submitted work. EHW reports grants from National Institutes of Health-National Institute of Environmental Health Sciences, during the conduct of the study; and non-financial support from Servier Labs, outside the submitted work. JS reports grants from USEPA, during the conduct of the study; and serving as Expert Consultant for The US Department of Justice in a lawsuit against Volkswagen for violating emissions limits, Expert Consultant for Natural Resources Defense Fund in a lawsuit against coal-burning power plant for violating emissions limits. BAC reports grants from National Institutes of Health, grants from USEPA, outside the submitted work. UH reports grants from National Heart, Lung, and Blood Institute-Framingham Heart Study, during the conduct of the study; grants from Kowa Company, grants from American College of Radiology Imaging Network, personal fees from the American Heart Association, outside the submitted work. MAM reports grants from USEPA, grants from National Institutes of Health, during the conduct of the study; grants from USEPA, grants from National Institutes of Health, outside the submitted work.

Ethics approval Institutional Review Boards of Boston University Medical Center, Beth Israel Deaconess Medical Center and the Massachusetts General Hospital.

Provenance and peer review Not commissioned; externally peer reviewed. Data sharing statement No additional data are available. 
Open Access This is an Open Access article distributed in accordance with the Creative Commons Attribution Non Commercial (CC BY-NC 4.0) license, which permits others to distribute, remix, adapt, build upon this work noncommercially, and license their derivative works on different terms, provided the original work is properly cited and the use is non-commercial. See: http:// creativecommons.org/licenses/by-nc/4.0/

\section{REFERENCES}

1. Brook RD, Rajagopalan S, Pope CA III, et al. Particulate matter air pollution and cardiovascular disease: An update to the scientific statement from the American Heart Association. Circulation 2010;121:2331-78.

2. Pope CA III, Dockery DW. Health effects of fine particulate air pollution: lines that connect. J Air Waste Manag Assoc 2006;56:709-42.

3. Hoek G, Krishnan RM, Beelen R, et al. Long-term air pollution exposure and cardio- respiratory mortality: a review. Environ Health 2013;12:43.

4. Dockery DW, Pope CA III, Xu X, et al. An association between air pollution and mortality in six U.S. cities. N Engl J Med 1993;329:1753-9.

5. Cesaroni G, Forastiere F, Stafoggia M, et al. Long term exposure to ambient air pollution and incidence of acute coronary events: prospective cohort study and meta-analysis in 11 European cohorts from the ESCAPE Project. BMJ 2014;348:f7412.

6. Suwa T, Hogg JC, Quinlan KB, et al. Particulate air pollution induces progression of atherosclerosis. J Am Coll Cardiol 2002;39:935-42.

7. Sun $\mathrm{Q}$, Wang $\mathrm{A}$, Jin $\mathrm{X}$, et al. Long-term air pollution exposure and acceleration of atherosclerosis and vascular inflammation in an animal model. JAMA 2005;294:3003-10.

8. Miller MR, McLean SG, Duffin R, et al. Diesel exhaust particulate increases the size and complexity of lesions in atherosclerotic mice. Part Fibre Toxicol 2013;10:61.

9. Hoffmann B, Moebus S, Möhlenkamp S, et al. Residential exposure to traffic is associated with coronary atherosclerosis. Circulation 2007; 116:489-96.

10. Diez Roux AV, Auchincloss AH, Franklin TG, et al. Long-term exposure to ambient particulate matter and prevalence of subclinical atherosclerosis in the Multi-Ethnic Study of Atherosclerosis Am J Epidemiol 2008;167:667-75.

11. Wilker EH, Mittleman MA, Coull BA, et al. Long-term exposure to black carbon and carotid intima-media thickness: the Normative Aging Study. Environ Health Perspect 2013;121:1061-7.

12. Rivera $M$, Basagaña $X$, Aguilera I, et al. Association between long-term exposure to traffic-related air pollution and subclinical atherosclerosis: the REGICOR study. Environ Health Perspect 2013;121:223-30.

13. Kälsch H, Hennig F, Moebus $\mathrm{S}$, et al. Are air pollution and traffic noise independently associated with atherosclerosis: the Heinz Nixdorf Recall Study. Eur Heart J 2013;35:853-60.

14. Provost EB, Madhloum N, Int Panis L, et al. Carotid intima-media thickness, a marker of subclinical atherosclerosis, and particulate air pollution exposure: the meta-analytical evidence. PLOS ONE 2015;10:e0127014.

15. Liu X, Lian H, Ruan $\mathrm{Y}$, et al. Association of exposure to particular matter and carotid intima-media thickness: a systematic review and meta-analysis. Int J Environ Res Public Health 2015;12: $12924-40$

16. Kaufman JD, Adar SD, Barr RG, et al. Association between air pollution and coronary artery calcification within six metropolitan areas in the USA (the Multi-Ethnic Study of Atherosclerosis and Air Pollution): a longitudinal cohort study. Lancet 2016;338:696-704.

17. Brook RD, Rajagopalan S. Particulate matter air pollution and atherosclerosis. Curr Atheroscler Rep 2010;12:291-300.

18. Campen MJ, Lund A, Rosenfeld M. Mechanisms linking trafficrelated air pollution and atherosclerosis. Curr Opin Pulm Med 2012;18:155-60.

19. Miller MR. The role of oxidative stress in the cardiovascular actions of particulate air pollution. Biochem Soc Trans 2014;42:1006-11.

20. Crouse DL, Peters PA, van Donkelaar A, et al. Risk of nonaccidental and cardiovascular mortality in relation to long-term exposure to low concentrations of fine particulate matter: a Canadian national-level cohort study. Environ Health Perspect 2012:120:708-14.

21. Zeger SL, Dominici F, McDermott A, et al. Mortality in the Medicare population and chronic exposure to fine particulate air pollution in urban centers (2000-2005). Environ Health Perspect 2008;116:1614-19.
22. Shi L, Zanobetti A, Kloog I, et al. Low-Concentration PM2.5 and mortality: estimating acute and chronic effects in a population-based study. Environ Health Perspect 2016;124:46-52.

23. Tonne C, Melly S, Mittleman M, et al. A case-control analysis of exposure to traffic and acute myocardial infarction. Environ Health Perspect 2007;115:53-7.

24. Wilker EH, Mostofsky E, Lue SH, et al. Residential Proximity to High-Traffic Roadways and Poststroke Mortality. J Stroke Cerebrovasc Dis 2013;22:e366-e72.

25. Lue SH, Wellenius GA, Wilker EH, et al. Residential proximity to major roadways and renal function. $J$ Epidemiol Community Health 2013;67:629-34.

26. Madrigano J, Kloog I, Goldberg R, et al. Long-term exposure to PM2.5 and incidence of acute myocardial infarction. Environ Health Perspect 2013;121:192-6.

27. Wilker EH, Mittleman MA, Coull BA, et al. Long-term exposure to black carbon and carotid intima-media thickness: the normative aging study. Environ Health Perspect 2013;121: 1061-7.

28. Allen RW, Criqui MH, Diez Roux AV, et al. Fine particulate matter air pollution, proximity to traffic, and aortic atherosclerosis. Epidemiology 2009;20:254-64.

29. Chuang ML, Massaro JM, Levitzky YS, et al. Prevalence and distribution of abdominal aortic calcium by gender and age group in a community-based cohort (from the Framingham Heart Study). Am J Cardiol 2012;110:891-6.

30. Allison MA, Criqui MH, Wright CM. Patterns and risk factors for systemic calcified atherosclerosis. Arterioscler Thromb Vasc Biol 2004;24:331-6.

31. Wong ND, Lopez VA, Allison M, et al. Abdominal aortic calcium and multi-site atherosclerosis: the Multiethnic Study of Atherosclerosis. Atherosclerosis 2011;214:436-41.

32. Rivera JJ, Nasir K, Katz R, et al. Relationship of thoracic aortic calcium to coronary calcium and its progression (from the Multi-Ethnic Study of Atherosclerosis [MESA]). Am J Cardiol 2009;103:1562-7.

33. Takasu J, Budoff MJ, O'Brien KD, et al. Relationship between coronary artery and descending thoracic aortic calcification as detected by computed tomography: the Multi-Ethnic Study of Atherosclerosis. Atherosclerosis 2009;204:440-6.

34. Gonçalves FB, Voûte MT, Hoeks SE, et al. Calcification of the abdominal aorta as an independent predictor of cardiovascular events: a meta-analysis. Heart 2012;98:988-94

35. Levitzky YS, Cupples LA, Murabito JM, et al. Prediction of intermittent claudication, ischemic stroke, and other cardiovascular disease by detection of abdominal aortic calcific deposits by plain lumbar radiographs. Am J Cardiol 2008;101:326-31.

36. Wilson PW, Kauppila LI, O'Donnell CJ, et al. Abdominal aortic calcific deposits are an important predictor of vascular morbidity and mortality. Circulation 2001:103:1529-34.

37. Witteman JC, Kannel WB, Wolf PA, et al. Aortic calcified plaques and cardiovascular disease (the Framingham Study). Am J Cardiol 1990;66:1060-4.

38. Witteman JC, Kok FJ, van Saase JL, et al. Aortic calcification as a predictor of cardiovascular mortality. Lancet 1986;2:1120-2.

39. Hermann DM, Lehmann N, Gronewold J, et al. Thoracic aortic calcification is associated with incident stroke in the general population in addition to established risk factors. Eur Heart J Cardiovasc Imaging 2015;16:684-90.

40. Budoff MJ, Nasir K, Katz R, et al. Thoracic aortic calcification and coronary heart disease events: the multi-ethnic study of atherosclerosis (MESA). Atherosclerosis 2011;215:196-202.

41. Eisen A, Tenenbaum A, Koren-Morag N, et al. Calcification of the thoracic aorta as detected by spiral computed tomography among stable angina pectoris patients: association with cardiovascular events and death. Circulation 2008:118:1328-34.

42. Dorans KS, Wilker EH, Li W, et al. Residential proximity to major roads, exposure to fine particulate matter, and coronary artery calcium: the Framingham Heart Study. Arterioscler Thromb Vasc Biol 2016;36:1679-85.

43. Kannel WB, Feinleib M, McNamara PM, et al. An investigation of coronary heart disease in families. The Framingham Offspring Study. Am J Epidemiol 1979;110:281-90.

44. Splansky GL, Corey D, Yang Q, et al. The Third Generation cohort of The National Heart, Lung, and Blood Institute's Framingham Heart Study: design, recruitment, and initial examination. $\mathrm{Am}$ $J$ Epidemiol 2007;165:1328-35.

45. Hoffmann U, Massaro JM, Fox CS, et al. Defining normal distributions of coronary artery calcium in women and men (from the Framingham Heart Study). Am J Cardiol 2008;102:1136-41, 41 e1. 
46. Cupples LA DAR, Kiely D. Section 34. Some risk factors related to the annual incidence of cardiovascular disease and death using pooled repeated Biennial measurements. In: Kannel WW, Garrison RJ. eds. The Framingham Study. An Epidemiological Investigation of Cardiovascular Disease. Bethesda, MD: National Heart, Lung, and Blood Institute, 1987:1-26.

47. Wilker $\mathrm{EH}$, Ljungman PL, Rice MB, et al. Relation of long-term exposure to air pollution to brachial artery flow-mediated dilation and reactive hyperemia. Am J Cardiol 2014;113:2057-63.

48. Zhou Y, Levy Jl. Factors influencing the spatial extent of mobile source air pollution impacts: a meta-analysis. BMC Public Health 2007;7:89.

49. Rosenbloom JI, Wilker EH, Mukamal KJ, et al. Residential proximity to major roadway and 10-year all-cause mortality after myocardial infarction. Circulation 2012;125:2197-203.

50. Kloog I, Chudnovsky AA, Just AC, et al. A new hybrid spatio-temporal model for estimating daily multi-year PM2.5 concentrations across northeastern USA using high resolution aerosol optical depth data. Atmospheric Environment 2014;95:581-90.

51. Rice MB, Ljungman PL, Wilker EH, et al. Long-term exposure to traffic emissions and fine particulate matter and lung function decline in the Framingham Heart Study. Am J Respir Crit Care Med 2015;191:656-64.

52. Miller KA, Siscovick DS, Sheppard L, et al. Long-term exposure to air pollution and incidence of cardiovascular events in women. N Engl J Med 2007;356:447-58.

53. Wilker EH, Preis SR, Beiser AS, et al. Long-term exposure to fine particulate matter, residential proximity to major roads and measures of brain structure. Stroke 2015;46:1161-6.

54. Agatston AS, Janowitz WR, Hildner FJ, et al. Quantification of coronary artery calcium using ultrafast computed tomography. J Am Coll Cardiol 1990;15:827-32.

55. Efron B. Nonparametric Standard Errors and Confidence Intervals. Canadian J Stat 1981;9:139-58.
56. Onuma OK, Pencina KM, Massaro JM, et al. Abstract P325: association of risk factors and extra-coronary Atherosclerosis with Progression of Coronary Artery Calcification in the Framingham Heart Study. Circulation 2014(129: AP325).

57. Kannel WB, Sorlie P. Some health benefits of physical activity. The Framingham Study. Arch Intern Med 1979;139:857-61.

58. Goff DC Jr, Lloyd-Jones DM, Bennett G, et al. 2013 ACC/AHA guideline on the assessment of cardiovascular risk: a report of the American College of Cardiology/American Heart Association Task Force on Practice Guidelines. Circulation 2014;129(Suppl 2):S49-73.

59. Harrell FE. Regression modeling strategies : with applications to linear models, logistic regression, and survival analysis. New York: Springer, 2001.

60. Buis M. POSTRCSPLINE: Stata module containining post-estimation commands for models using a restricted cubic spline, 2009.

61. Tsao CW, Pencina KM, Massaro JM, et al. Cross-sectional relations of arterial stiffness, pressure pulsatility, wave reflection, and arterial calcification. Arterioscler Thromb Vasc Biol 2014;34:2495-500.

62. Budoff MJ, Achenbach S, Blumenthal RS, et al. Assessment of coronary artery disease by cardiac computed tomography a scientific statement from the American Heart Association Committee on Cardiovascular Imaging and Intervention, Council on Cardiovascular Radiology and Intervention, and Committee on Cardiac Imaging, Council on Clinical Cardiology. Circulation 2006;114:1761-91.

63. Pletcher MJ, Tice JA, Pignone M, et al. Using the coronary artery calcium score to predict coronary heart disease events: a systematic review and meta-analysis. Arch Intern Med 2004;164:1285-92.

64. Stein JH, Korcarz CE, Hurst RT, et al. Use of carotid ultrasound to identify subclinical vascular disease and evaluate cardiovascular disease risk: a consensus statement from the American Society of Echocardiography Carotid Intima-Media Thickness Task Force. Endorsed by the Society for Vascular Medicine. J Am Soc Echocardiogr 2008;21:93-111. quiz 89-90. 